Features of electron and phonon processes in $\mathrm{GaSb}-\mathrm{FeGa}_{1.3}$ eutectics

This content has been downloaded from IOPscience. Please scroll down to see the full text.

2003 J. Phys. D: Appl. Phys. 362627

(http://iopscience.iop.org/0022-3727/36/21/005)

View the table of contents for this issue, or go to the journal homepage for more

Download details:

IP Address: 193.140.249.2

This content was downloaded on $26 / 05 / 2016$ at $14: 12$

Please note that terms and conditions apply. 


\title{
Features of electron and phonon processes in GaSb-FeGa1.3 eutectics
}

\author{
M I Aliyev ${ }^{1}$, A A Khalilova ${ }^{1}$, D H Arasly ${ }^{1}$, R N Rahimov ${ }^{1,4}$, \\ $M$ Tanoglu ${ }^{2}$ and L Ozyuzer ${ }^{3}$ \\ ${ }^{1}$ Institute of Physics of the Azerbaijan, National Academy of Sciences, 33 H.Javid av., \\ Az-1143, Baku, Azerbaijan \\ ${ }^{2}$ Department of Mechanical Engineering, İzmir Institute of Technology, Gulbahce campus, \\ 35437, Urla, Izmir, Turkey \\ ${ }^{3}$ Department of Physics, İzmir Institute of Technology, Gulbahce campus, 35437, Urla, \\ Izmir, Turkey \\ E-mail: rashad@physics.ab.az
}

Received 11 July 2003

Published 15 October 2003

Online at stacks.iop.org/JPhysD/36/2627

\begin{abstract}
Eutectic alloys of $\mathrm{GaSb}-\mathrm{FeGa}_{1.3}$ were prepared by the vertical Bridgman method. A microstructure with the needle-shaped metallic $\mathrm{FeGa}_{1.3}$ phase oriented in a specific direction and uniformly distributed within the $\mathrm{GaSb}$ matrix was obtained. In $\mathrm{GaSb}-\mathrm{FeGa}_{1.3}$ eutectics, the electrical and thermal conductivity, thermal diffusivity, thermoelectric power and Hall coefficients were investigated in a wide temperature range. These properties were measured at different mutual directions of current, thermal flow, magnetic field and metal phase inclusions. The influence of metallic inclusions on these properties was revealed and the distinctive characteristics of electron and phonon processes were established.
\end{abstract}

\section{Introduction}

Eutectic alloys of the semiconductor-metal type where the metallic phase exists in the form of needles are one of the best material models of heterogeneous semiconductors. The advantage of such compositions is the combination of semiconductor and metal properties. Furthermore, there is a possibility of tailoring these properties through the composition and expanding the functional range of the materials to fulfill the needs of advanced technology. At present, eutectic alloys based on $\mathrm{A}^{3} \mathrm{~B}^{5}$ compounds find a wide area of applications for creation of galvanomagnetic, thermomagnetic, photothermomagnetic and strain sensitive transducers [1-5]. A number of works have been dedicated to experimental study to develop eutectic alloys with uniformly distributed parallel metal needles [1-9]. Among these eutectics, the InSb based alloys were most extensively studied. Studying the physical properties of eutectic compositions of the semiconductor-metal type is of interest due to the distinctive features of electron and phonon processes in heterogeneous semiconductors. In this paper, electric and thermal properties of the $\mathrm{GaSb}-\mathrm{FeGa}_{1.3}$ compositions as a

4 Author to whom any correspondence should be addressed. function of needle orientation between 80 and $500 \mathrm{~K}$ were investigated.

\section{Experimental}

Semiconductor matrix elements (GaSb) with a hole concentration of $1.7 \times 10^{17} \mathrm{~cm}^{-3}$ were obtained by alloying the related components in stoichiometric quantities and refined by the method of horizontal recrystallization. Eutectic alloys were prepared by alloying $92.1 \mathrm{wt} \% \mathrm{GaSb}$ and $7.9 \mathrm{wt} \% \mathrm{FeGa}_{1.3}$ using the vertical Bridgman method [10]. The rate of growth of the crystallization front was about $1 \mathrm{~mm} \mathrm{~min}^{-1}$. Employing this technique, a structure with the needle-shaped metallic $\mathrm{FeGa}_{1.3}$ phase oriented in a specific direction and uniformly distributed within the GaSb matrix was obtained. Samples were prepared in two orientations of $\mathrm{FeGa}_{1.3}$ metallic needles: oriented parallel (along the length of the samples) and perpendicular (lateral to the samples) to the longitudinal axis of the samples.

Specimens for microstructural investigations were prepared by polishing with traditional techniques and etching in $\mathrm{CP}-4\left(\mathrm{HCl}+\mathrm{HF}+\mathrm{CH}_{3} \mathrm{COOH}-2: 11: 2\right)$ after washing in alcohol. A scanning electron microscope (SEM), model Philips $^{\mathrm{TM}}$, and an energy dispersive $\mathrm{x}$-ray spectroscope (EDX), 
model $\mathrm{EDAX}^{\mathrm{TM}}$, was used to characterize the morphology of the specimens and to obtain qualitative information on the elemental composition of the samples, respectively. The accelerating voltage during EDX analysis was $15 \mathrm{kV}$. Thermogravimetric measurements were done using a thermal analysis device, Derivatograph. Samples for electric and thermal measurements were prepared in parallelepiped form with size $(2 \times 4 \times 12) \mathrm{cm}^{3}$. On both the lateral sides of the samples, four contact probes were attached to measure the electrical conductivity $(\sigma)$, thermal power $(\alpha)$ and Hall coefficient $(R)$ using the compensation method. The thermal conductivity $(K)$ was measured by the absolute stationary method, and the thermal diffusivity $(a)$ was measured by the light flash heating method.

\section{Results and discussion}

Figure 1 shows SEM micrograph of GaSb-FeGa 1.3 showing cross sections of the samples along the longitudinal and lateral directions of the needle-shaped $\mathrm{FeGa}_{1.3}$ phase. The oriented needles were found to be about $1 \mu \mathrm{m}$ in diameter, $20-150 \mu \mathrm{m}$ in length and with $N=3.3 \times 10^{4} \mathrm{~mm}^{-2}$ density. Characteristic $x$-rays were generated during EDX analysis. The $\mathrm{x}$-ray spectra obtained from the needle and matrix phases along the longitudinal and lateral directions are illustrated in figure 2, with the SEM images showing the location of the investigations. The elemental compositions are also shown in the same figures. It was found that the metallic inclusions contain mainly $\mathrm{Fe}$ and $\mathrm{Ga}$, with a minor amount of $\mathrm{Sb}$ along the longitudinal and the lateral directions. Similarly, the matrix contains $\mathrm{Sb}$ (about $60 \mathrm{wt} \%$ ) and $\mathrm{Ga}$ (about $40 \mathrm{wt} \%$ ) along both the directions. Figures 3 and 4 show elemental maps of $\mathrm{Fe}$, $\mathrm{Ga}$ and $\mathrm{Sb}$ from the cross sections along the longitudinal and lateral direction of the needle phases, respectively. The image on the upper left is the SEM image showing the topography of the mapped region. In the elemental maps, each specific colour indicates an element and black colour indicates the absence of this element. In the specific map, the colours red, green and yellow indicate $\mathrm{Fe}(\mathrm{K}), \mathrm{Ga}(\mathrm{K})$ and $\mathrm{Sb}(\mathrm{L})$, respectively. The results confirm the formation of the needleshaped $\mathrm{FeGa}_{1.3}$ phase oriented in a specific direction and

(a)

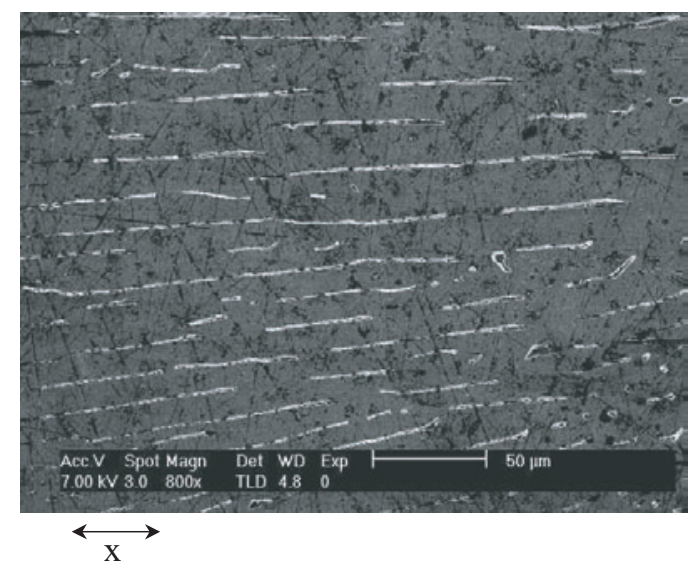

uniformly distributed within the GaSb matrix. The elemental maps also reveal the formation of inclusions within the matrix with distinct boundaries. The distribution of the elements illustrated in the maps is in general in accordance with the elemental compositions shown in figure 2. However, Sb within the metallic inclusions is not visible in elemental maps due to its minor concentration in these phases.

Figure 5 shows the thermogravimetry measurement results of the $\mathrm{GaSb}-\mathrm{FeGa}_{1.3}$ samples. The major endothermic peak at about $1003 \mathrm{~K}$ is associated with melting of the alloy. The initial and final temperatures of melting were determined as $963 \mathrm{~K}$ and $1003 \mathrm{~K}$, respectively. Also, the heat $(Q)$, entropy $(\Delta S)$ and activation energy $(E)$ of melting were calculated as $32.4 \mathrm{~J} \mathrm{~g}^{-1}, 6.17 \mathrm{~kJ} \mathrm{~K}^{-1} \mathrm{~mol}^{-1}$ and $79.8 \mathrm{~kJ} \mathrm{~mol}^{-1}$ from the graphs. Moreover, the reaction at about $1173 \mathrm{~K}$ is associated with oxidation of the alloy and this is referred to as the oxidation temperature $\left(T_{\mathrm{ox}}\right)$.

The temperature dependence of electrical conductivity and the Hall coefficient at different mutual directions of current $(I)$, magnetic field $(B)$ and the needle orientation axis $(x)$ are given in figures $6(a)$ and $(b)$. In the case of $I \perp x$, the $\sigma$ values and their dependence on temperature, $\sigma(\mathrm{T})$ for the eutectic alloy is similar to that for the matrix material, GaSb, with the corresponding concentration. For $I \| x$, an increase in electrical conductivity takes places due to the short-circuiting effect of metallic inclusions.

The short-circuiting effect of metallic inclusions $\left(\mathrm{FeGa}_{1,3}\right)$ also has some influences on the temperature dependence of the Hall coefficient (figure 6(b)). In contrast to the GaSb matrix, which strongly depends on temperature, in eutectic alloys the Hall coefficient has the anisotropy depending on mutual directions of current, magnetic field and metallic inclusions and it becomes appreciable at low temperatures. In the case of $I \perp x \perp B$, the Hall field is oriented parallel with metallic inclusions, which results in the short-circuit. The minimum value of the Hall coefficient in the case of $I \| x \perp B$ is related to the current running mainly through metallic inclusions. The value of $R$ is a maximum when the metallic needles in the $\mathrm{GaSb}$ phase are orientated perpendicular to the current direction and parallel to the magnetic field $(I \perp x \| B)$ and the dependence behaviour $R(T)$ is approximately the same as for the matrix. (b)

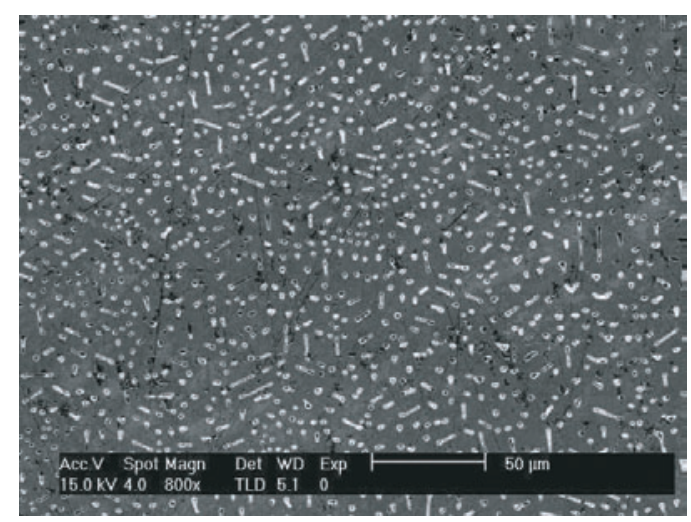

Figure 1. SEM micrographs of GaSb-FeGa ${ }_{1.3}$ showing cross sections of the samples along the $(a)$ longitudinal and $(b)$ lateral directions of the needle-shaped $\mathrm{FeGa}_{1.3}$ phase. 
(a)
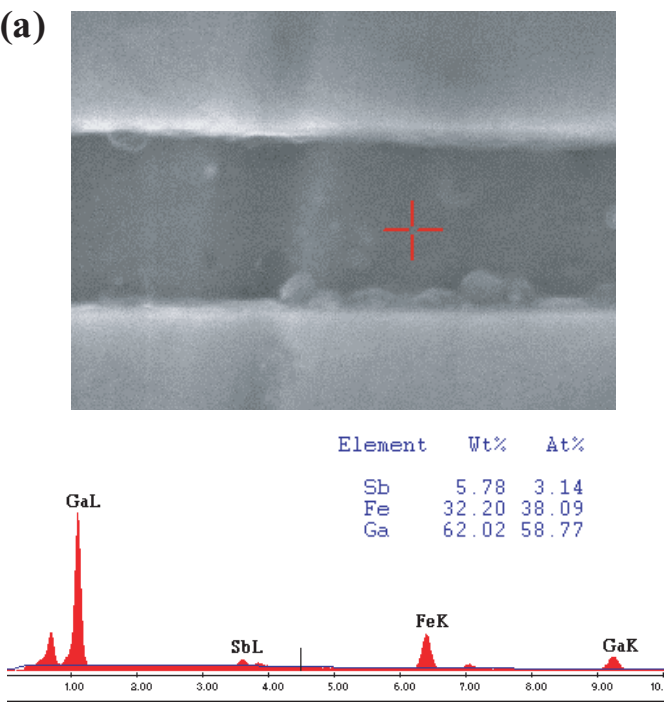

(b)
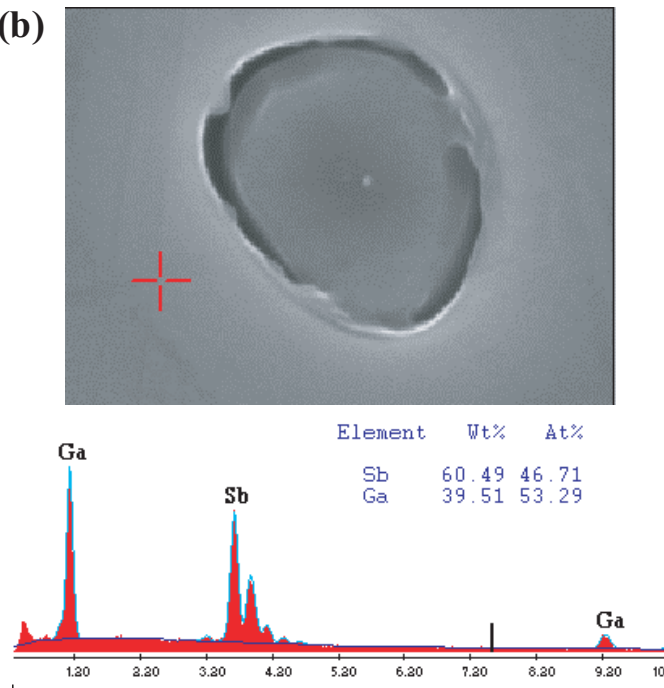

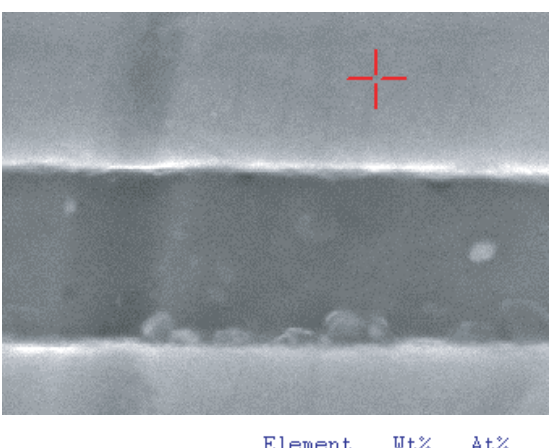

$\begin{array}{lll}\mathrm{Sb} & 57.58 & 43.74 \\ \mathrm{Ga} & 42.42 & 56.26\end{array}$
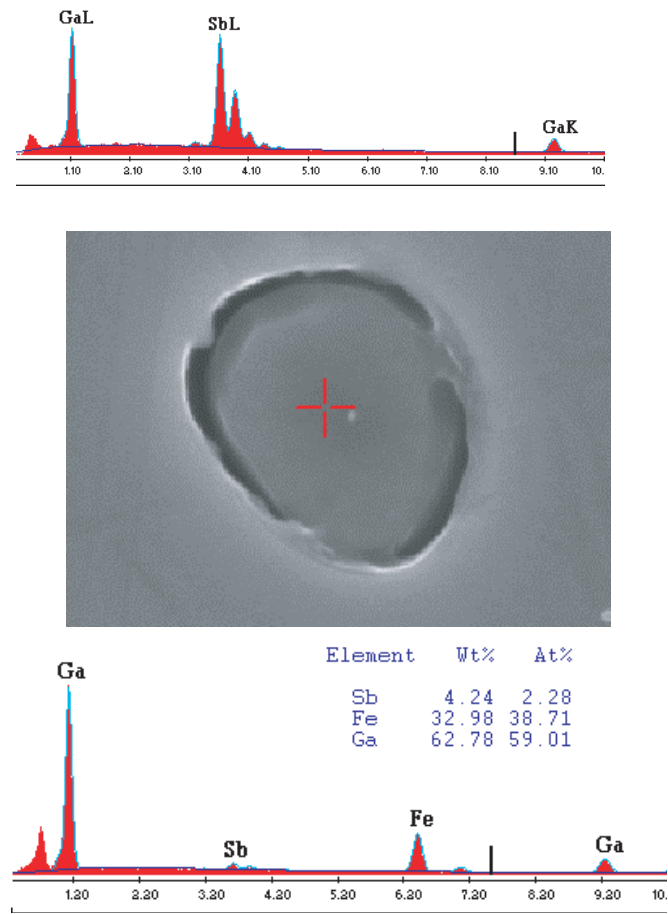

Figure 2. X-ray spectra of GaSb-FeGa ${ }_{1.3}$ obtained with SEM-EDX from the needle and matrix phases along the $(a)$ longitudinal and (b) lateral directions of the specimens.

The problem of determining the effective conductivity (electrical and thermal) of composite materials has been discussed for a long time. The effective conductivity is determined using the perturbation theory [11] when the degree of heterogeneity of a system is small, using the percolation theory when the medium is highly heterogeneous [12-16] and using the effective medium theory in the intermediate case [17-22]. Experimental results for the electric conductivity and other coefficients of kinetic effects obtained in this work are basically interpreted as qualitative within the framework of the effective medium theory. Different models have been suggested and a number of expressions to calculate effective parameters in eutectic compositions have been obtained [21]. These expressions include matrix and metal inclusion parameters and volume fraction of metal phase. Computations of generalized conductivity of heterogeneous systems were carried out by Odelevskiy [22]. It was shown that depending on the phase resistance value and current direction, either phase conductivities or resistances are added. For interpretation of the experimental data obtained from InSb-Sb eutectic alloys,
Liebman and Miller [6] have suggested a model. Taking continuity of the thermal flow into account, Leonov et al [7] applied the model for InSb-NiSb eutectic alloys. According to Liebman and Miller [6] and Leonov et al [7], the eutectic structure can be represented as two parallel conductors in the case of $I \| x$ and as a combination of parallel and series conductors in the case of $I \perp x$. So, electrical conductivity parallel to the metal inclusions $\left(\sigma_{\|}\right)$and perpendicular to the metal inclusions $\left(\sigma_{\perp}\right)$ may be determined by the following formulae:

$$
\begin{gathered}
\sigma_{\|}=\sigma_{1} \frac{1}{1+c}+\sigma_{2} \frac{c}{1+c} \\
\sigma_{\perp}=\frac{\left(\sigma_{1}-\sigma_{2}\right)(1-\sqrt{c /(1+c)})+\sigma_{1} \sqrt{(1+c) / c}}{1+\left(\sigma_{2} / \sigma_{1}\right)(\sqrt{(1+c) / c}-1)},
\end{gathered}
$$

where, $\sigma_{1}$ and $\sigma_{2}$ are the electric conductivity of the matrix and the inclusions and $c$ is the volume fraction of the metal inclusions.

Since there are no data available for $\mathrm{FeGa}_{1.3}$, qualitative calculation of total electric conductivity for the GaSb-FeGa $\mathrm{Fa}_{1.3}$ 

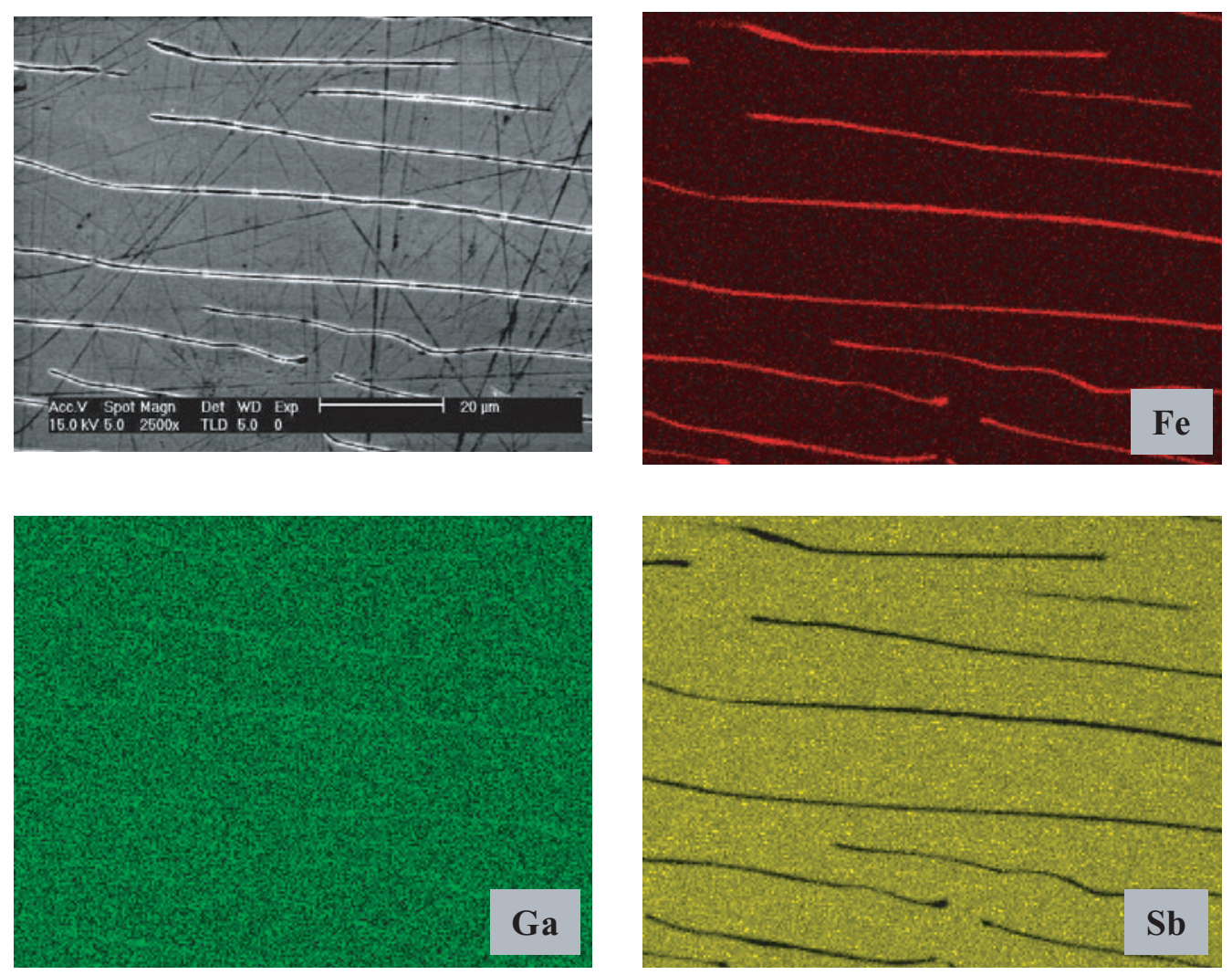

Figure 3. Elemental maps of $\mathrm{Fe}, \mathrm{Ga}$ and $\mathrm{Sb}$ obtained with EDX from $\mathrm{GaSb}-\mathrm{FeGa}_{1.3}$ along the longitudinal direction of the needle-shaped $\mathrm{FeGa}_{1.3}$ phase.
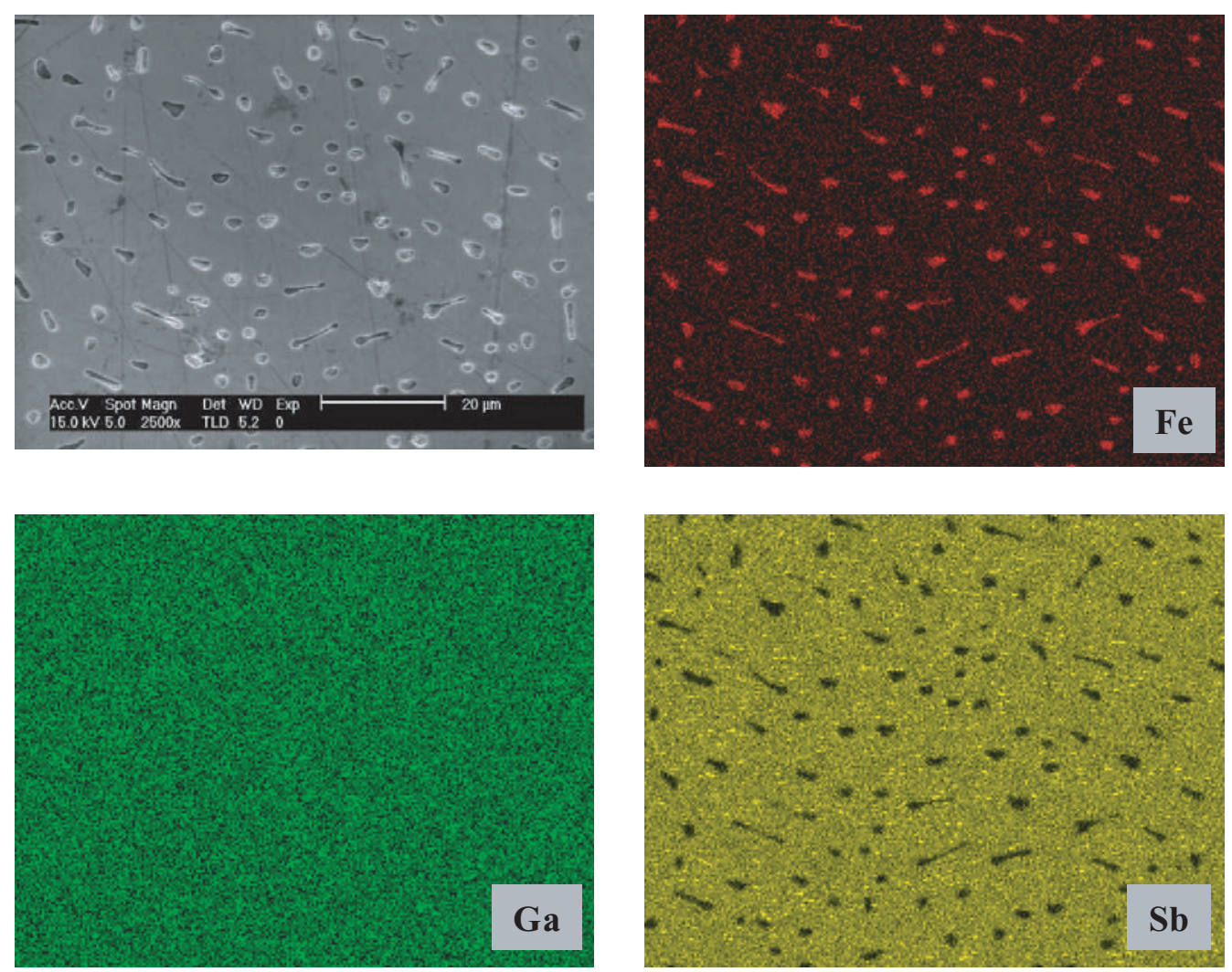

Figure 4. Elemental maps of $\mathrm{Fe}, \mathrm{Ga}$ and $\mathrm{Sb}$ obtained with $\mathrm{EDX}$ from $\mathrm{GaSb}-\mathrm{FeGa}_{1.3}$ along the lateral direction of the needle-shaped $\mathrm{FeGa} \mathrm{I}_{1.3}$ phase. 


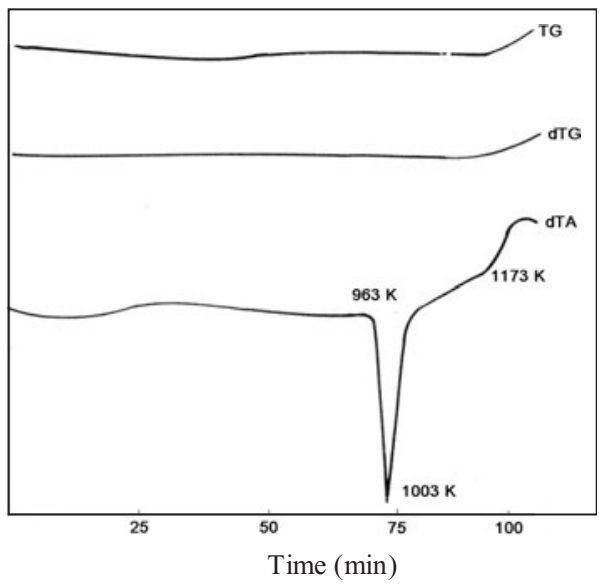

Figure 5. Thermogravimetry results of the $\mathrm{GaSb}-\mathrm{FeGa}_{1.3}$ system.
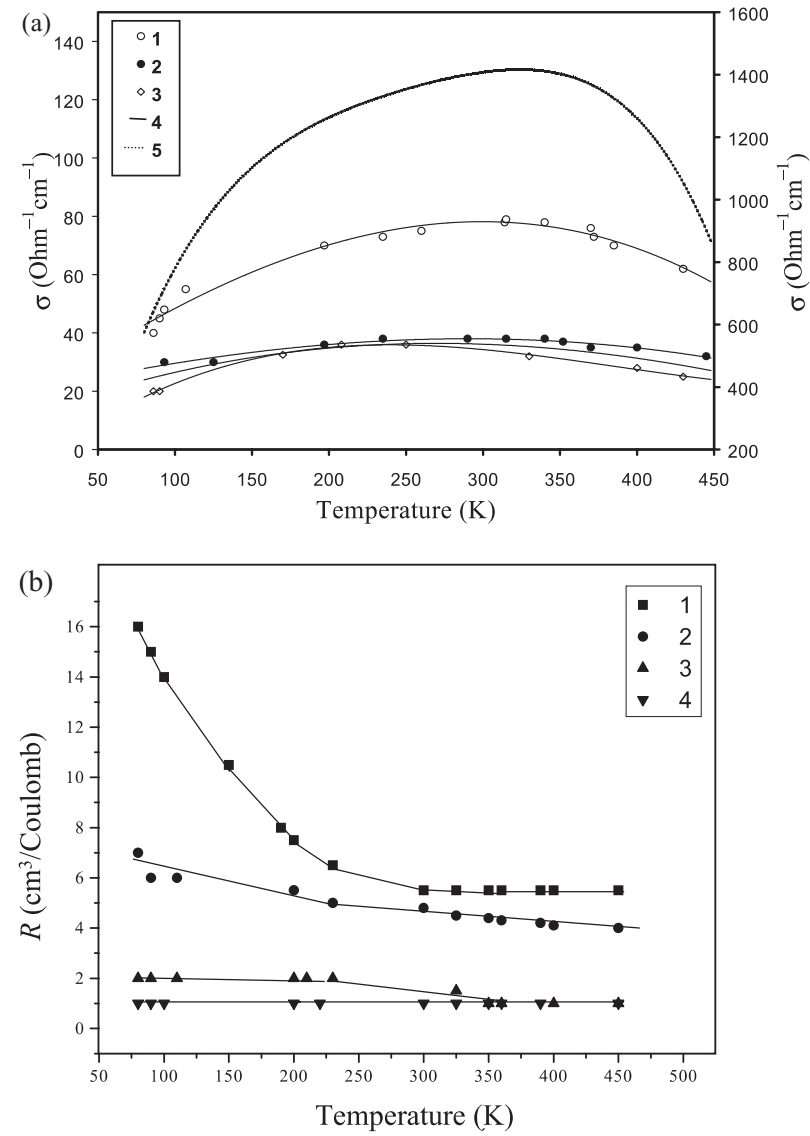

Figure 6. (a) Temperature dependence of electric conductivity for $\mathrm{GaSb}-\mathrm{FeGa}_{1.3}$ at $I \| x(1), \mathrm{GaSb}-\mathrm{FeGa}_{1.3}$ at $I \perp x$ (2), GaSb $\left(p=2 \times 10^{18} \mathrm{~cm}^{-3}\right)(3)$. The calculated curves for $\mathrm{GaSb}(4)$ and FeGa (5) are also given. (b) Temperature dependence of the Hall coefficient for $\mathrm{GaSb}$ (1), GaSb-FeGa ${ }_{1.3}$ at $I \perp x \| B$ (2), GaSb-FeGaa $\mathrm{Fa}_{1.3}$ at $I \| x \perp B$ (3) and GaSb-FeGa ${ }_{1.3}$ at $I \perp x \perp B$ (4).

eutectic has not been carried out. However, the electric conductivity of the matrix $\left(\sigma_{1}\right)$ and metallic inclusions $\left(\sigma_{2}\right)$ has been calculated by solving equations (1) and (2) on the basis of the electrical conductivity of the matrix and metallic inclusions using the volume fractions of the phases and experimental data on the electrical conductivity of the eutectic at different directions of current and needles. The calculated electric
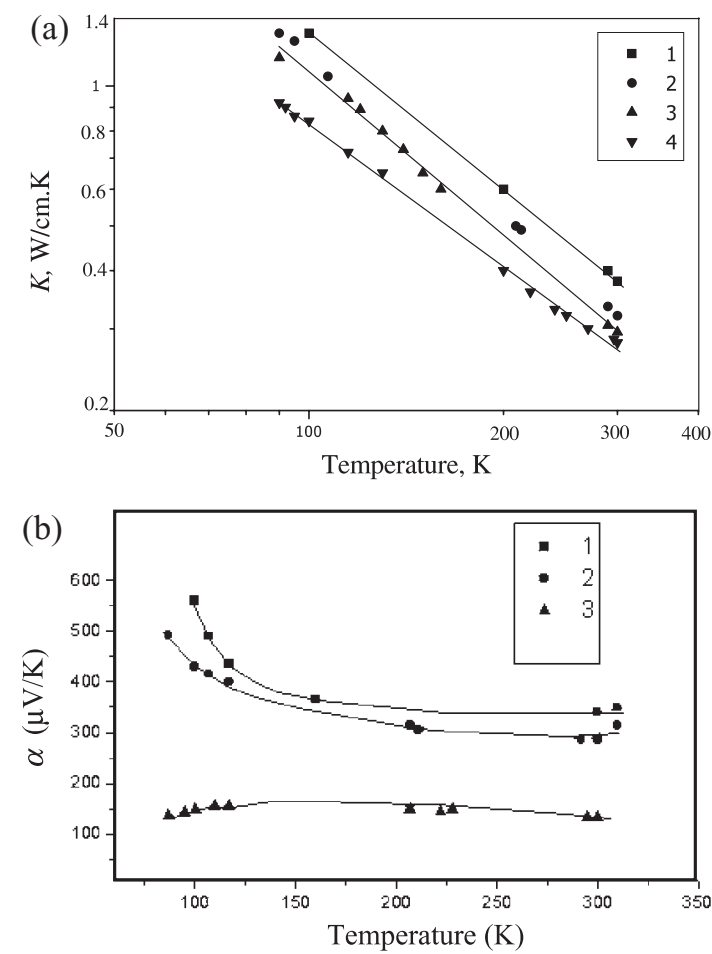

Figure 7. (a) Temperature dependence of total thermal conductivity for $\operatorname{GaSb}\left(p=1.7 \times 10^{17} \mathrm{~cm}^{-3}\right)(1), \mathrm{GaSb}\left(p=2.1 \times 10^{18} \mathrm{~cm}^{-3}\right)$ (2), GaSb-FeGa 13 at $W \| x$ (3) and $\mathrm{GaSb}-\mathrm{FeGa}_{13}$ at $W \perp x$ (4). (b) Temperature dependence of thermoelectric power for $\mathrm{GaSb}$ $\left(p=2 \times 10^{18} \mathrm{~cm}^{-3}\right)$ based on Aliyev et al [25] (1), GaSb-FeGa ${ }_{1.3}$ at $W \perp x(2)$ and $\mathrm{GaSb}-\mathrm{FeGa}_{1.3}$ at $W \| x(3)$.

conductivity of $\mathrm{FeGa}_{1.3}$ inclusions is shown in figure 6(a) as a function of temperature and it is an order of magnitude greater than the matrix value.

The temperature dependence of the total thermal conductivity $(K)$ and thermoelectric power $(\alpha)$ of $\mathrm{GaSb}$ and $\mathrm{GaSb}-\mathrm{FeGa}_{1,3}$ with thermal flow $(W)$ parallel $(W \| x)$ and perpendicular $(W \perp x)$ to the orientation of the needles $(x)$ is given in figures $7(a)$ and $(b)$. As may be seen, the $K$ values exhibit anisotropy, the magnitude of which decreases with increasing temperature. The ratio $K_{\|} / K_{\perp}$ at $80 \mathrm{~K}$ is equal to 1.3 and the $K_{\|}$and $K_{\perp}$ values approach each other with increasing temperature, where $K_{\|}$and $K_{\perp}$ refer to thermal conductivity parallel and perpendicular to thermal flow, respectively. The thermal conductivity due to the charge carriers in $\mathrm{GaSb}-\mathrm{FeGa}_{1.3}$ was calculated on the basis of the Weidemann-Franz law as $4 \%$ of $K_{\text {total }}$ and it is acceptable that the heat is generally transported by phonons. The thermal conductivity in heterogeneous systems with cylindrical inclusions may be expressed by the following formulae [22]:

$$
\begin{gathered}
K_{\|}=K_{1}\left[1+\frac{c}{K_{1} /\left(K_{2}-K_{1}\right)}\right], \\
K_{\perp}=K_{1}\left(1+\frac{c}{(1-c) / 2+K_{1} /\left(K_{2}-K_{1}\right)}\right),
\end{gathered}
$$

where the indices ' 1 ' and ' 2 ' refer to the semiconductor and metal phases, respectively, and $c$ is the volume fraction of the metal inclusions. The influence of the inclusions on the thermal conductivity is negligible due to their low volume fraction. 
In spite of the minor contribution of the inclusions to heat transport in the $\mathrm{GaSb}-\mathrm{FeGa}_{1,3}$ eutectic, they may have a strong influence on the phonon scattering event. The similar temperature dependences of the thermal conductivities of the eutectics (at $W \| x)$ and GaSb $\left(p=2 \times 10^{18} \mathrm{~cm}^{-3}\right)$ (figure 7(a), curves 2 and 3 ) indicate that the metallic inclusions in those directions do not essentially contribute to the thermal resistance. As the diameter of the needles $(d \sim 1 \mu \mathrm{m})$ is much less than their length $(L \sim 100 \mu \mathrm{m})$, the additional thermal resistance in the case of $W \perp x$ is probably connected with the scattering of phonons on the cross boundaries of metal needles. Shortwave phonon scattering may also occur due to the matrix doping by $\mathrm{Fe}$ impurities that takes place during the $\mathrm{GaSb}-\mathrm{FeGa}_{1,3}$ synthesis. The thermal resistance caused by phonon scattering at point defects was calculated by Klemens' formula [23] as $0.4 \mathrm{~cm} \mathrm{~K} \mathrm{~W}^{-1}$ at $300 \mathrm{~K}$ and $0.23 \mathrm{~cm} \mathrm{~K} \mathrm{~W}^{-1}$ at $80 \mathrm{~K}$. Here, the appropriate parameters were taken for $\mathrm{GaSb}$ and only the change of density around the defects was taken into account. Because the shortwave phonons intensively scatter at impurities, the contribution of long-wave phonons to the thermal conductivity becomes significant.

The mean free path of phonons $(l)$ calculated on the basis of $l=K / 3 C V$, in which $C$ is the thermal capacity and $V$ is the speed of sound, is $0.1 \mu \mathrm{m}$ and $0.04 \mu \mathrm{m}$ at $80 \mathrm{~K}$ and $300 \mathrm{~K}$, respectively. However, the mean free path of long-wave phonons may be determined from the expression of London-Rumer and Simons [24] for the relaxation time $\tau^{-1}=(2 \pi / \rho)(2 \pi V / k T)^{4} q$. Here, $\rho$ is the density and $q$ is the wave vector of phonons. The mean free path of longwave phonons was calculated as $1.1 \mu \mathrm{m}$ and $4.1 \times 10^{-2} \mu \mathrm{m}$ at $80 \mathrm{~K}$ and at $300 \mathrm{~K}$, respectively. As may be seen, at low temperatures the mean free path of long-wave acoustic phonons is comparable with the diameter $(d=1 \mu \mathrm{m})$ of the metallic needle-shaped inclusions. We assume that the observed anisotropy of thermal conductivity is associated with the scattering of long-wave phonons at the boundaries between the semiconductor and metal phases for the $W \perp x$ orientation. The weakening of the hole-drag effect by phonons in the $\mathrm{GaSb}-\mathrm{FeGa}_{1.3}$ eutectic in comparison with $\mathrm{GaSb}$ of a similar concentration may also be related to the scattering of long-wave phonons on the inclusion boundaries. An anisotropy was also observed in the thermoelectric properties of the eutectic. For $W \| x$, the short-circuit leads to a decrease of thermal power $(\alpha)$. Note the temperature dependence of thermoelectric power at the $W \perp x$ orientation. As seen in figure 7(b), below $200 \mathrm{~K}, \alpha_{\perp}$ increases with decreasing temperature. Such a rise in thermal power can be due to the hole-drag by phonons as observed in homogeneous GaSb and reported by Aliyev et al [25]. It is known that when long-wave acoustic phonons fit the condition for $q \leqslant 2 k$ (where $q$ and $k$ are wave vectors of phonons and charge carriers at the Fermi level, respectively), the drag effect occurs. One must note that the increase in thermoelectric power for the eutectic alloys is smaller than that for GaSb with the charge carrier concentration close to the eutectic alloys (figure 7(b), curves 1 and 2) and it is related to long-wave phonon scattering at the inclusion boundaries, as in the case of the thermal conductivity at $W \perp x$.

The features that are typical for metal inclusions are also clearly seen in the temperature dependence of the thermal diffusivity of the eutectics as seen in figure 8 . In the case

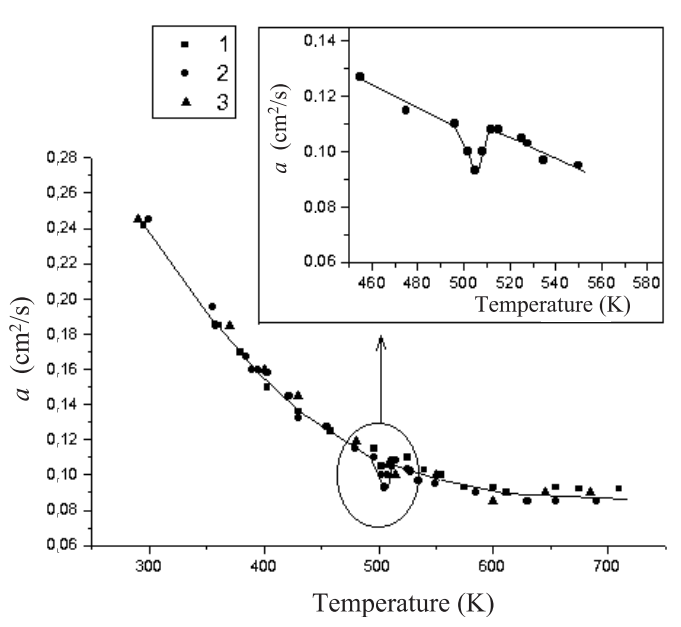

Figure 8. Temperature dependence of thermal diffusivity for $\mathrm{GaSb}$ $\left(p=2 \times 10^{18} \mathrm{~cm}^{-3}\right)(1), \mathrm{GaSb}-\mathrm{FeGa}_{1.3}$ at $W \| x(2)$ and GaSb-FeGa ${ }_{1.3}$ at $W \perp x(3)$.

of $W \| x$, the thermal diffusivity values exhibit a minimum at $T=505 \pm 5 \mathrm{~K}$ such that the peak value is much greater than the possible measurement errors. Since $\mathrm{GaSb}-\mathrm{FeGa}_{1.3}$ is ferromagnetic, this minimum appears to be associated with the transition from a ferromagnetic to a paramagnetic state. The magnetism of the $\mathrm{GaSb}-\mathrm{FeGa}_{1.3}$ eutectic is probably due to the presence of the $\mathrm{FeGa}_{1.3}$ inclusions and the temperature at which the peak occurs corresponds to the Curie temperature for this compound.

\section{Summary}

Investigation of the electric and thermal conductivity, Hall coefficient and thermoelectric power of $\mathrm{GaSb}-\mathrm{FeGa}_{1.3}$ at various mutual directions of current, magnetic field and temperature gradient and orientation of needle-shaped metal inclusions revealed an anisotropy of the kinetic coefficients due to the presence of a metal phase. It is established that the anisotropy of thermal conductivity and the weakening of the hole-drag effect by phonons in the $\mathrm{GaSb}-\mathrm{FeGa}_{1.3}$ eutectic are associated with the scattering of long-wave phonons at the metal inclusion boundaries. A minimum value of thermal diffusivity at temperatures about $505 \pm 5 \mathrm{~K}$ for the case where the thermal flow is directed parallel to the metallic needles may be related to the transition from the ferromagnetic to paramagnetic state.

\section{Acknowledgments}

The authors acknowledge the financial support from NATO under Collaborative Linkage Grant PST.CLGN 978434. Also, L.O. acknowledges the support from the Turkish Academy of Science in the framework of a Young Scientist Award (LO/TUBA-GEBIP/2002-1-17).

\section{References}

[1] Weiss H 1969 Structure and Application of Galvanomagnetic Devices (Oxford: Pergamon) 
[2] Paul B, Weiss H and Wilhelm M 1964 Solid State Electronics vol 7 (Oxford: Pergamon) p 835

[3] Uher C and Goldsmid H I 1972 J. Phys. D: Appl. Phys. 5 $1478-88$

[4] Sopovskaya M A and Smetannikova Y S 1987 Fizika i Tekhnika Poluprovodnikov (Semiconductors) 21 1242-6

[5] Aliyev M I, Isakov G I, Aliyev F Y and Eminzade A T 1989 Rep. Acad. Sci. USSR 306 583-6 (in Russian)

[6] Liebman W K and Miller E A 1963 J. Appl. Phys. 34 2653-9

[7] Leonov V V, Chunarev E N and Spektor Y E 1977 Physical-chemical process in heterogeneous system (in Russian) Krasnoyarsk 67

[8] Aliyev M I, Jabbarov R M, Arasly D H and Aliyeva M A 1973 Inorg. Mater. 7 427-8

[9] Aliyev M I, Abdinova S G and Aliyev S A 1972 Phys. Status Solidi a $957-8$

[10] Muller A and Wilhem M 1965 J. Phys. Chem. Solids 26 2029-35

[11] Herring C 1960 J. Appl. Phys. 31 107-22

[12] Shante V K S and Kirpatrick S 1971 Adv. Phys. 20 325-57

[13] Shklovskii B I 1972 Fizika i Tekhnika Poluprovodnikov (Semiconductors) 61197
[14] Rodriguez M E, Perez-Bueno J J, Zelaya-Angel O and Conzalez-Hernandez J 1998 Mater. Lett. 36 95-101

[15] Xue Q 2003 Physica B 325 195-8

[16] Sareni B, Krahenbuhi I and Beroual A 1997 J. Appl. Phys. 81 2375-83

[17] Bruggeman D A G 1935 Ann. Phys. (Leipzig) 24 636-79

[18] Kirkpatrick S 1973 Rev. Mod. Phys. 45 574-88

[19] Zallen R 1983 The Physics of Amorphous Solids (New York: Wiley)

[20] Doyle W T and Jacobs I S 1990 Phys. Rev. 42 9319-27

[21] Emetz Y P 1986 Electric Characteristics of Composite Materials with the Regular Structure (Kiev: Naukova Dumka)

[22] Odelevskiy B I 1951 J. Tech. Phys. 21 667-77 (in Russian)

[23] Szmulowicz F, Madarasz F L, Klemens P and Diller J 1989 J. Appl. Phys. 66 252-5

[24] Askerov B M 1994 Electron Transport Phenomena in Semiconductors (Singapore: World Scientific)

[25] Aliyev M I, Jafarov Z A and Aliyeva M A 1971 Fizika i Tekhnika Poluprovodnikov (Semiconductors) 5 337-9 